\title{
Automatic Modulation Classification Using Dense Memory Fusion Network
}

\author{
Anping $\mathrm{Li}^{1}$, Juanjuan Huang ${ }^{2}$, Xiaofei Zhang ${ }^{1}, \mathrm{Xu} \mathrm{Yang}^{{ }^{*}}$, Meiying Wei ${ }^{1}$ \\ \{lianping@srrc.org.cn¹, jjhuang@bupt.edu.cn², zhangxf@srrc.org.cn ${ }^{1}$, \\ yangxu@srrc.org.cn ${ }^{1}$, weimeiying@srrc.org.cn ${ }^{1}$ \}
}

The State Radio Monitorning Center, Beijing, P.R.China, 100037¹.

Key Laboratory of Universal Wireless Communications, Ministry of Education, Beijing University of Posts and Telecommunications, Beijing, P.R.China, $100876^{2}$.

\begin{abstract}
Automatic modulation classification (AMC), as a key technology of cognitive radio (CR), aims to identify the modulation format of the received signal. In this paper, we propose a novel dense memory fusion neural network(DMFN) based AMC method where grid constellation matrix (GCM) extracted from the received signals with low computational complexity are utilized as the input of DMFN. In DMFN, densnet with densely connected structures is designed to extract high representative feature of GCMs, the unit of long short-term memory (LSTM) and fully connected layer are used to make classification decisions. Extensive simulations demonstrate that DMFN yields significant performance gain and takes higher robustness comparing with other methods. In addition, DMFN based AMC scheme achieves $90 \%$ classification accuracy at $4 \mathrm{~dB}$ when the symbol length is set as 512, which illustrates its outstanding performance.
\end{abstract}

Keywords: Cognitive Radio, Automatic Modulation Classification, Deep Learning, Dense Memory Fusion Neural Network.

\section{Introduction}

With the rapid growth of mobile devices, the radio frequency (RF) spectrum resource become more scarce[1][2][3]. The development of radio technology is largely constrained by the shortage of RF spectrum resource. To alleviate the spectrum shortage, cognitive radio (CR)[4], Cognitive Radio proposes a dynamic spectrum access method, which can dynamically access the idle band to avoid interference by perceptually the state of the target band[5]. It shows that there is a need for improved spectrum sensing and signal identification algorithms to enable sensors and radios to detect and identify spectrum users and interference in the widest possible range, thereby improving the signal-to-noise ratio (SNR). In order to ensure the normal usage of the spectrum in CR, automatic modulation classification (AMC), which servers as an intermediate step between signal detection and demodulation, is a promising key technology to identify the modulation formats of the target signals corrupted by noise and interference. AMC is widely used in military and civil communication fields, such as software radio, cognitive radio, spectrum detection and management, signal detection, adaptive modulation transmission, threat analysis, interference identification, signal authentication, electronic reconnaissance, non-cooperative communication, etc.[6]. It plays an important role in intelligent signal analysis. 
In general, AMC methods can be roughly divided into two different types, which are the likelihood-based (LB) and feature-based (FB) approaches[7][8]. LB method take AMC as a hypothesis testing problem, which can get the optimal solution under Bayesian sense by minimizing the probability of misclassification. However, when the receiving signal appears parameters of an unknown probability distribution, the complexity of the method increases and the robustness decreases, which greatly limits its application in practice[9][10] . Though FB methods are sub-optimal methods, it is easier to apply in practical implementations than the former method because of its low computational complexity and less prior knowledge required. So this paper is going to improve the performance of FB methods to get more classification accuracy gain.

The FB-based AMC method can be roughly divided into two steps. The first step is to extract statistical features from the received signal. The second step is to use the extracted feature parameters to design a classifier for classification. In recent years, many researchers use some novel features to improve the performance of the FB method. Smith et al. in [11] used the high-order cumulant feature as the input of a fully connected neural network to implement the AMC method, however, the computational complexity of higher-order cumulants is high. The extracted characteristic feature include amplitude-phase information, and signal constellation representation. Plenty of novel neural networks are utilized to process those features, such as Alexnet [12], Resnet [13]. However, both of them can not satisfy the requirement of high performance at low SNR. Some other researchers pay attention to change the loss function to constrained neural network to converges. Sai Huang et al. use contrastive loss [14], compressive loss [15] to enhance intra-class aggregation and inter-class separability, which may make it harder to converge for networks. Moreover, part of his effort was devoted to identifying overlapped sources by using multi-gene genetic programming with structural risk minimization rinciple [16].

In this paper, we propose a novel dense memory fusion neural network (DMFN) based AMC method. Firstly, we utilize grid constellation matrix projection to generated GCMs, which contains the constellation information of the received signal and discards some redundant information. Secondly, a dual model fusion network structure is designed to learn the deep information of GCMs and improve AMC performance. Thirdly, simulations are conducted to verify the performance and robustness of proposed DMFN based AMC method.

\section{Problem statement}

\subsection{Signal model}

Assume that the radio frequency signal is received by the signal antenna system and transformed to baseband signal. Therefore, a general expression for the complex envelope of the received baseband signal is given by

$$
\begin{gathered}
y(n)=s(n)+g(n), \\
s(n)=A e^{j 2 \pi f_{0} T_{n}+j \theta_{n}} \sum_{i=-\infty}^{\infty} x(l) h\left(n T-l T+\varepsilon_{T} T\right),
\end{gathered}
$$


where $A$ and ${ }^{x(l)}$ represent the unknown amplitude and symbol sequence, respectively. denotes the residual channel effects and is invariant during the AMC process. $f_{o}$ is the frequency offset, and $\theta_{n}$ represents the phase jitter. $T_{\text {and }} \varepsilon_{T}$ are the symbol spacing and timing errors, respectively.

$g(n)$ is the additive white Gaussian noise. Suppose that the average power of each symbol is normalized, the signal-to-noise ratio (SNR) is formulated by

$$
S N R=\frac{E\left\{|s(n)|^{2}\right\}}{E\left\{|g(n)|^{2}\right\}}
$$

\subsection{System model}

Fig. 1 presents the modulation classification Architecture of this paper, which can be roughly divided into to modules, i. e., feature extraction module and LSTM-based classification module. Firstly, the raw IQ signal received by the antenna receiver should go through the feature extraction module, which can extract GCM features different from the raw IQ feature and the traditional constellation. Secondly, we utilize the GCM as the input of the classification, which is composed of DenseNet and LSTM. Finally, we got the hypothesis $H_{k}$ of this AMC issue, in which ${ }^{k}$ is set as 5 .

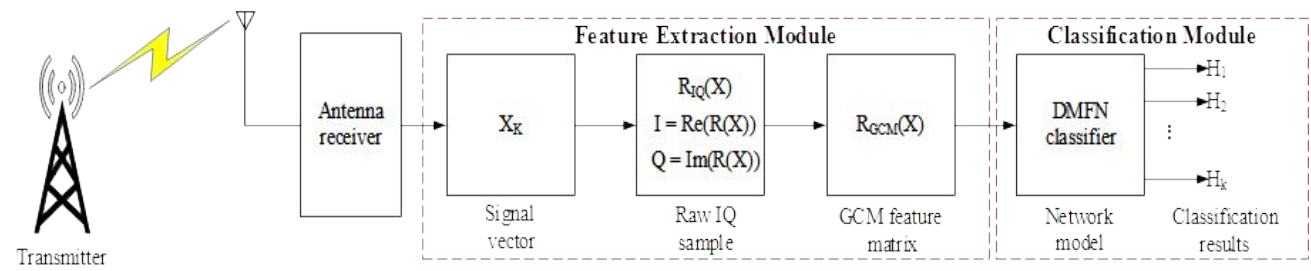

Fig. 1. Dense memory fusion neural network based AMC scheme.

\section{DMFN based modulation classification}

\subsection{Grid constellation matrix}

Considering that the received signal can be separated by two part: the imaginary part and the real part [17], we often project the raw "IQ" signal into cartesian coordinate system through mapping the I component to the $\mathrm{X}$-axis, and the $\mathrm{Q}$ component to the $\mathrm{Y}$-axis. I, Q components denote the imaginary and real part of the received complex signal, respectively. Regular constellation images (RC) are generated by mapping the $\mathrm{I}, \mathrm{Q}$ data into scattering points on the complex plane.

In order to facilitate the network to learn effective representations from the signal, the received signal are transformed into GCM as the input of the network. Firstly, we pre-draw the $M \times N$ grid on the RC. Secondly, we calculate the number of sample points that fall in each 
grid, and finally, after all the sample points are calculated, we normalize the $M \times N$ grid, resulting in a grayscale matrix image that represents the density of the constellation map.

In this paper, the predetermined size of the grid $M \times N$ are set to 40 , both. Some example of GCM for five modulation formats versus SNRs are given in Fig. 2. The highlight pixels in the image indicate that signal symbols are clustered in these area.

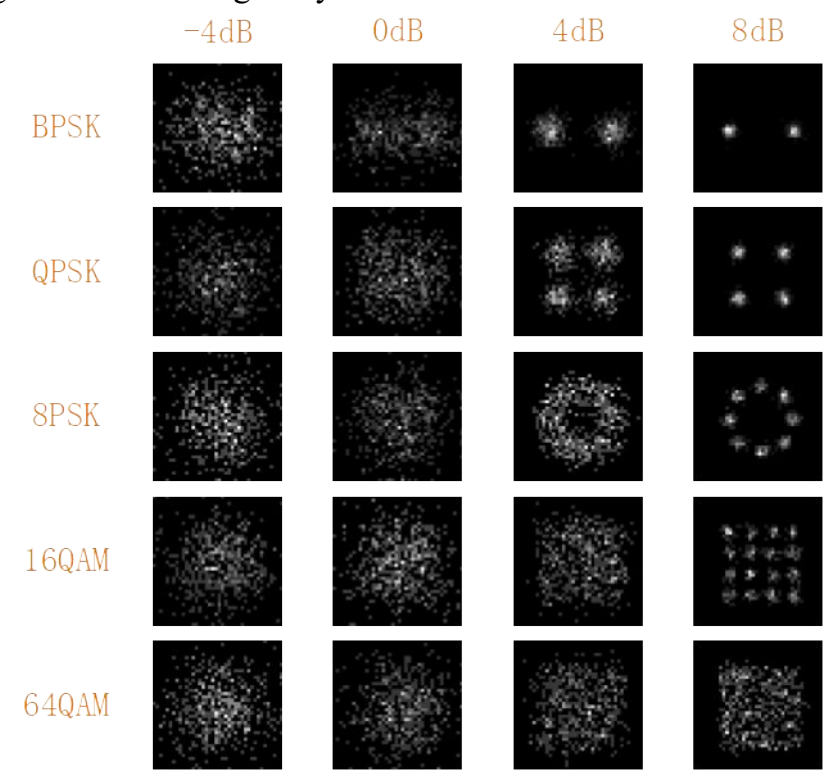

Fig. 2. GCMs for five modulation formats with different SNRs.

\subsection{Structure of DMFN}

In order to explore deep representations and recognize the signal format. DMFN, a datadriven automatic modulation classification structure is designed. As is shown in Fig., the DMFN mainly consist of two modules, the feature extraction module and the classification module. The feature extraction module extracts the deep representations from the raw GCM images. The classification module utilizes the deep high-dimensional representations to perform the final classification decision. As the GCM is quite different with the pictures of actual physical environment. Therefore, the DMFN is different with the deep learning networks using in computer vision field. Firstly, We set the convolutional kernel size as $3 \times 3$ to actualize sparse connectivity. Secondly, we utilize the LSTM to transform the highdimensional feature into one-dimensional feature using as the input of the last fully connected layer.

In the feature extraction module, the first $3 \times 3$ convolution is utilized to pre-process the raw GCM features and obtain the preliminary representations. The max-pooling performs down-sampling to reduce the size of the preliminary representations. Next, in order to incorporate efficiency and accuracy, we adopt four dense blocks to extract the underlying high-dimensional features. The four dense blocks have the similar structure. Take dense block 1 as example, it contain six units, one convolution and one pooling. The six unit make up the densely connections, each unit connects with all previous units to reuse the feature and strengthen feature propagation. The convolution after six units is used as the transformation to 
connect the dense blocks. Besides, rectified linear unit (Relu) activation function in the convolution is able to enhance the nonlinearity of the DMFN. The last part of dense block 1 is pooling, which is utilized to enhance the sparsity of the feature generated from the former convolution and reduce the number of network parameters.

After the feature extraction, we get the high-dimensional feature which can represent the raw signal format. However, the high-dimensional feature is unable to perform the finally classification directly. Hence, we use the classification module to reduce the dimension and improve the computation efficiency. The classification module consists of LSTM layer and fully connected layer, we first use the LSTM layer to transform the high-dimensional feature into one-dimensional feature. Then, fully connected layer output the unified units whose number is equal to the modulation formats. the value of the unified units are utilized to conduct the finally classification.

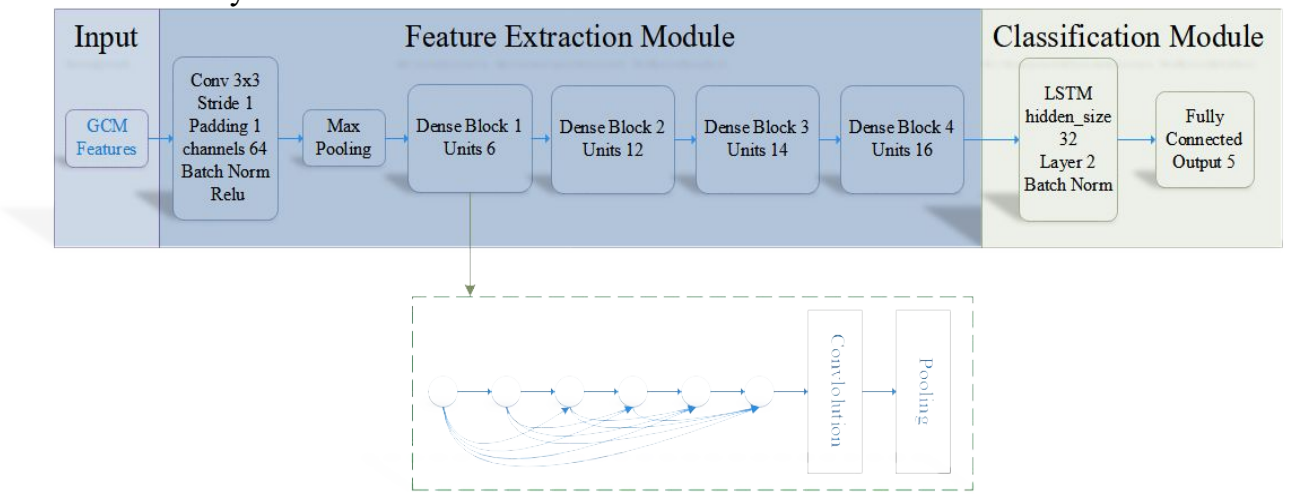

Fig. 3. The structure of DMFN.

\subsection{Loss function}

The most widely used classification loss function in multi-classification problem, crossentropy loss, is presented as follows:

$$
\begin{aligned}
& J_{c}(\mathbf{w})=-\frac{1}{N} \sum_{i=1}^{N} \log p\left(y_{i} \mid x_{i} ; \mathbf{w}\right) \\
& =-\frac{1}{N} \sum_{i=1}^{N} \log \prod_{j=1}^{n}\left(\frac{e^{\pi_{j} x_{j}+b_{n}}}{\sum_{j=1}^{n} e^{\pi_{j}^{\gamma} x_{j}}}\right)^{1(y-j)} \\
& =-\frac{1}{N} \sum_{i=1}^{N} \sum_{j=1}^{n} \log \left(\frac{e^{W_{j}^{2} x_{j}+b_{y}}}{\sum_{j=1}^{n} e^{\pi_{j}^{2} x+b_{j}}}\right)^{1(y-j)}
\end{aligned}
$$

Where ${ }^{1(\cdot)}$ is the indicator function, and $x_{i} \in \mathrm{R}^{d}$ denotes the deep feature of the ${ }^{i}$-th sample, belonging to the $y_{i}$-th class. The embedding feature dimension $d$ is set to 32 in this paper. 
$W_{j} \in \mathrm{R}^{d}$ denotes the ${ }^{j}$-th column of the weight $W \in \mathrm{R}^{d \times n}$ and $b_{j} \in \mathrm{R}^{*}$ is the bias term. The batch size and the class number are ${ }^{N}$ and ${ }^{n}$, respectively.

In order to prevent the network weights from taking extremely large values and overfitting, we add a regularization term to the cross-entropy loss function. So the loss function is given by:

$$
\begin{aligned}
J(\mathbf{w}) & =J_{c}(\mathbf{w})+J_{i}(\mathbf{w}) \\
& =J_{c}(\mathbf{w})+\alpha \frac{1}{N} \sum_{i=1}^{N} \sum_{j=!}^{n}\left\|W_{j}^{(i)}\right\|_{2}
\end{aligned}
$$

Where \|\|$\|$ denotes the Euclidean norm. ${ }^{W_{j}^{(i)} \in \mathrm{R}^{d} \text { denotes the }}{ }^{j}$-th column of the weight $W \in \mathrm{R}^{\alpha \times \infty}$ of the ${ }^{i}$-th sample and $\alpha$ is the coefficient of the regularization term, which is specifies by the user.

We update the weight $\mathrm{w}$ utilizing mini-batch stochastic gradient descent and error back propagation algorithm until the loss converges to a constant, and then get the optimal parameter ${ }^{*}$ of DMFN, which is given by

$$
\mathbf{w}^{*}=\arg \min _{\mathbf{w}} J(\mathbf{w})
$$

\section{Simulation}

In this section, extensive simulations are conducted to illustrate the superiority and offset robustness of the proposed DMFN. An open source dataset platform, RadioML (https://github.com/radioML), is used to generate the modulated signal for fair comparison. We utilize the RadioMl platform to generate the modulation signals and simulate each modulation format as SNR ranges from $-6 \mathrm{~dB}$ to $14 \mathrm{~dB}$ with a step of $2 \mathrm{~dB}$. The candidate modulation set is $\mathbf{M}=$ \{BPSK, QPSK, 8PSK, 16QAM, 64QAM $\}$. Ten thousand samples for each modulation format at certain SNR are used to train and verify the performance of proposed $\mathrm{AMC}$ scheme, and one thousand samples to test. In the training process, the Monte Carlo trails are used to calculate the probability of correctly classifying ${ }^{P_{x}}$, which is given as follows

$$
P_{c c}=\sum_{k=1}^{K} P\left(\hat{H}=H_{k} \mid H_{k}\right) P\left(H_{k}\right), H_{k} \in M
$$


Where $P\left(H_{k}\right)$ denotes the prior probability of modulation format ${ }^{H_{k}}$ and we assume that the prior probabilityof each modulation format is equal to ${ }^{\frac{1}{5}} . P\left(\hat{H}=H_{k} \mid H_{k}\right)$ is the probability that the modulation format is correctly estimated as $H_{k}$.

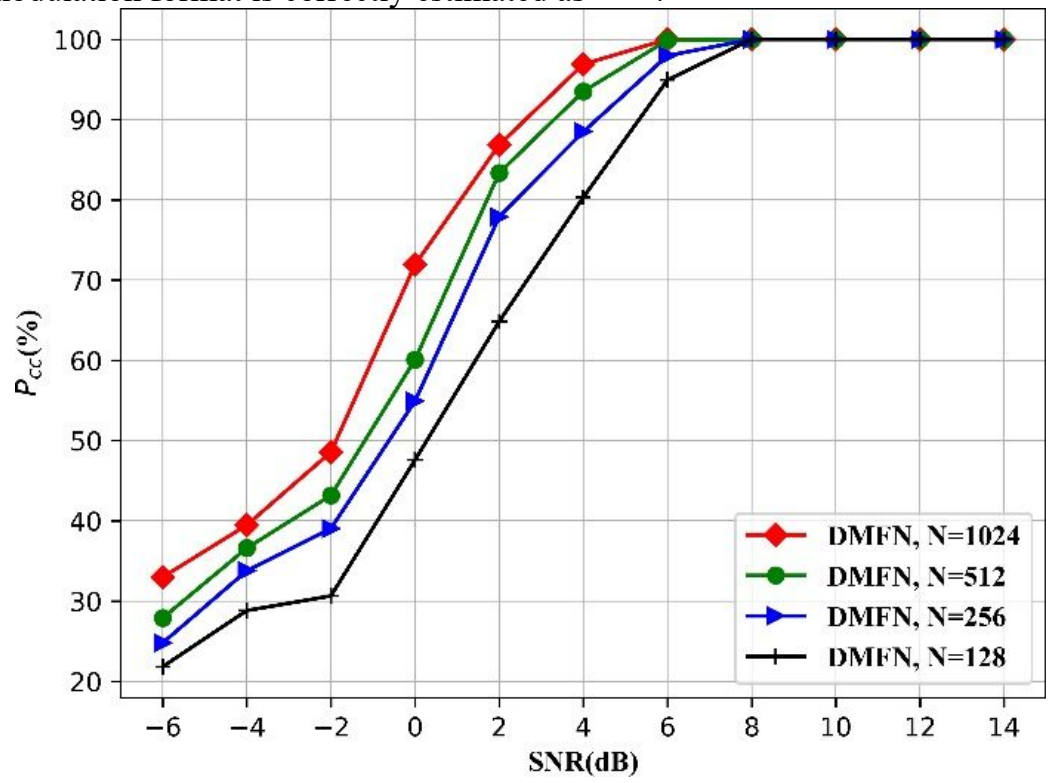

Fig. 4. Classification accuracy of DMFN versus symbol length.

Fig. 4 shows the classification performance of DMFN versus SNR with different symbol lengths. It is obvious that better classification performance is achieved by using more signal symbols. The classification accuracy reaches $94.94 \%$ when the SNR is $6 \mathrm{~dB}$ and the symbol length is 128 . 


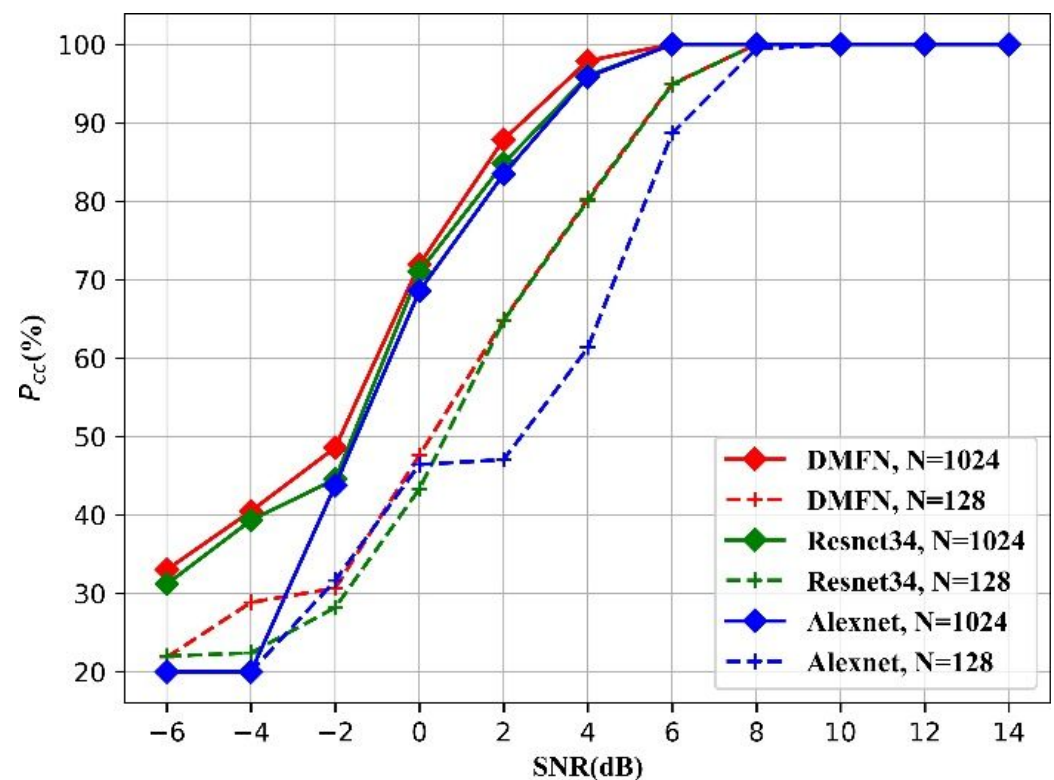

Fig. 5. Classification accuracy comparison among DMFN and the other AMC methods

Fig. 5 illustrates that the performance of DMFN is better than the other two AMC methods, i.e. Resnet[18], Alexnet[19], under the same symbol length and SNR. Those two deep learning neural network structures are selected to conduct comparison. We slightly modified the number of neurons in the final fully connected layer from the original 1000 to the 5, which is the number of modulation format. As is shown in Fig. 5, given the same symbol length, for Resnet34 and Alexnet, the former is better than the latter overall. However, Both of them are slightly insufficient compared with DMFN. The performance gain yielded by DMFN deep learning model can be explained by the combination of densenet and LSTM structure.

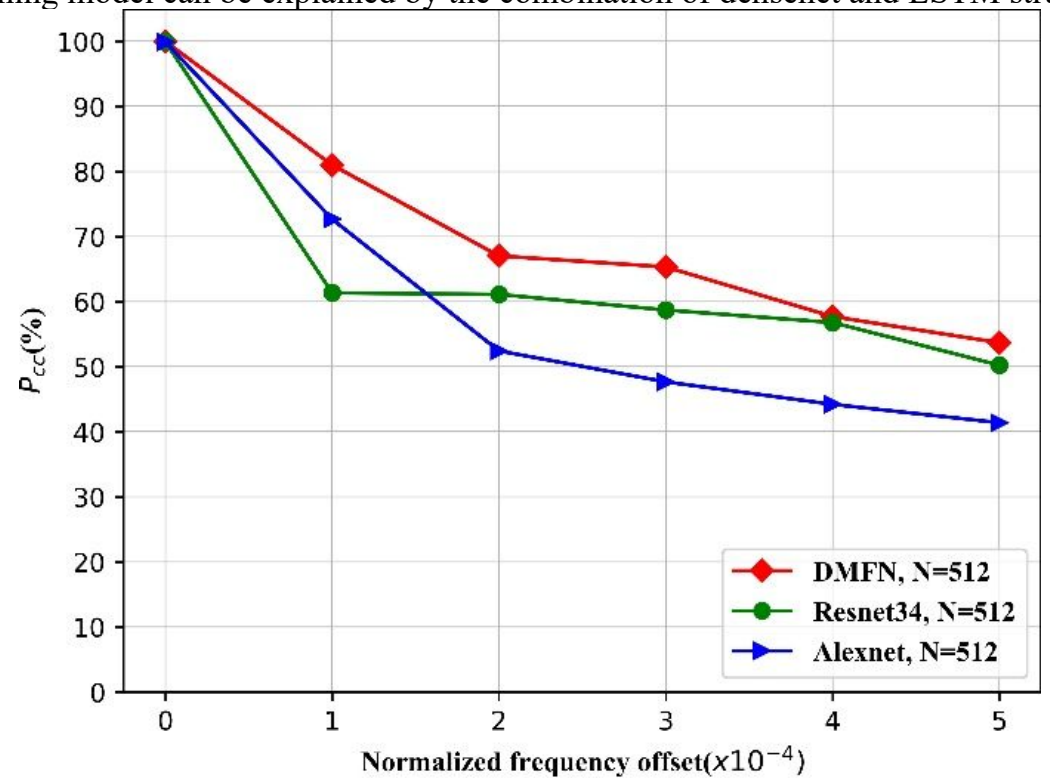


Fig. 6. The robustness of DMFN and the other AMC methods.

Fig. 6 compares the robustness of proposed DMFN, Resnet34, Alexnet. The robustness of DMFN based AMC scheme versus normalized frequency offset with symbol length $N=512$ is depicted. The symbol length and SNR are fixed at 512 and $6 \mathrm{~dB}$, respectively. The range of frequency offset is set from 0 to $5 \times 10^{-4}$ with a step of $10^{-4}$. It can be observed that the frequency offset severely degrades the classification performance and DMFN performs best of all, proving its robustness to frequency offset.

\section{Conclusions}

In this paper, we proposed a GCM based AMC method named DMFN. Firstly, GCM are utilized as the initial input of the network. Secondly, a densnet construction is conducted to extract distinguishable feature from GCMs and a LSTM based classifier is designed to make classification decisions. Finally, extensive simulations are designed to verify the superiority and robustness of DMFN compared with other existing methods.

Acknowledgement. This work was supported in part by the National Key Research and Development Program of China under Grant (2018YFF0301202, 2019YFB1804400), in part by the National Natural Science Foundation of China under Grant (61801052, 61227801), and in part by the Beijing Natural Science Foundation under Grants (4202046). 


\section{References}

[1] Gao, Yue, et al:: Scalable and reliable IoT enabled by dynamic spectrum management for M2M in LTE-A. vol. 3, no. 6, pp. 1135-1145. IEEE Internet of Things Journal, (2016)

[2] Lunden, Jarmo, Visa Koivunen, and H. Vincent Poor.: Spectrum exploration and exploitation for cognitive radio: Recent advances. vol. 32, no. 3, pp. 123-140. IEEE signal processing magazine, (2015)

[3] Ma, Yuan, et al:: Reliable and efficient sub-Nyquist wideband spectrum sensing in cooperative cognitive radio networks. vol.34, no. 10, pp. 2750-2762. IEEE Journal on Selected Areas in Communications, (2016)

[4] J. J. Meng, W. Yin, H. Li and et al.: Collaborative spectrum sensing from sparse observations in cognitive radio networks. vol.29, no. 2, pp 327-337. IEEE Journal on Selected Areas in Communications, (2011)

[5] Y. Ma, Y. Gao, A. Carvallaro and et al:: Sparsity Independent SubNyquist Rate Wideband Spectrum Sensing on Real-time Signals. vol. 66, no. 10, pp. 8784-8797. IEEE Trans. Veh. Technol, (2017)

[6] S. Huang, Y. Yao, Z. Wei, Z. Feng, and P. Zhang.: Automatic modulation classification of overlapped sources using multiple cumulants. vol. 66, no. 7, pp. 6089-6101. IEEE Trans. Veh. Technol, (2016)

[7] O. Dobre.: Signal identification for emerging intelligent radios: classical problems and new challenges. vol. 18, no. 2, pp. 11-18. IEEE Instrum. Meas. Mag, (2015)

[8] O. A. Dobre, A. Abdi, Y. Bar-Ness, and W. Su.: Survey of automatic modulation classification techniques: classical approaches and new trends. vol. 1, no. 2, pp. 137-156. IET communications, (2007)

[9] S. Majhi, R. Gupta, W. Xiang and et al.: Hypothesis and Feature Based Blind Modulation Classification for Linearly Modulated Signals. vol. 66, no. 12, pp. 11057-11069. IEEE Trans. Veh. Technol, (2017)

[10] W. Wei and J. M. Mendel.: Maximum-likelihood classification fordigital amplitude-phase modulations. vol. 48, no. 2, pp. 189-193. IEEE Transactions on Communications (2000)

[11] A. Smith, M. Evans, and J. Downey.: Modulation classification of satellite communication signals using cumulants and neural networks. Proc. IEEE CCAA, Cleveland, USA, Jun. 2017.

[12] Krizhevsky A, Sutskever I, Hinton G E.: Imagenet classification with deep convolutional neural networks. Advances in neural information processing systems Harrahs and Harveys, Lake Tahoe, USA, Curran Associates, Inc:1097-1105, (2012)

[13] He, Kaiming, et al.: Deep residual learning for image recognition. Proceedings of the IEEE conference on computer vision and pattern recognition. (2016)

[14] S. Huang, Y. Jiang, Y. Gao, Z. Feng and P. Zhang.: Automatic modulation classification using contrastive fully convolutional network. IEEE Wireless Commun. Letters. (2019)

[15] S. Huang, Y. Jiang, X. Qin et al.: Automatic Modulation Classification of Overlapped Sources Using Multi-Gene Genetic Programming With Structural Risk Minimization Principle. IEEE Access. pp. 48827-48839 (2018)

[16] S. Huang, L. Chai and Z. N. Li et al.: Automatic modulation classification using compressive convolutional neural network. IEEE Access., vol. 7, no. 1, pp. 79636-79643 (2019)

[17] Wang, Fen, Yongchao Wang, and Xi Chen.: Graphic constellations and DBN based automatic modulation classification. 2017 IEEE 85th Vehicular Technology Conference (VTC Spring), IEEE, (2017)

[18] He K, Zhang X, Ren S, et al.: Deep residual learning for image recognition. Proceedings of the IEEE conference on computer vision and pattern recognition. Las Vegas, America:770-778, (2016)

[19] Krizhevsky A, Sutskever I Hinton G E.: Imagenet classification with deep convolutional neural networks. Advances in neural information processing systems. Harrahs and Harveys, Lake Tahoe, USA, Curran Associates, Inc:1097-1105, (2012) 